\title{
PITCH-ANGLE SCATTERING OF COMETARY IONS: COMPUTER SIMULATIONS
}

\author{
S. Peter Gary \\ Los Alamos National Laboratory \\ Ronald H. Miller \\ University of Michigan \\ Dan Winske \\ Los Alamos National Laboratory
}

\begin{abstract}
Pitch-angle evolution of newborn cometary ions is studied by means of one-dimensional electromagnetic hylbrid computer simulations of homogeneous plasmas. Newborn ions are injected into the simulations at a constant rate, with a velocity relative to the solar wind which makes an angle $\alpha$ with respect to the ambient magnetic field. The simulations are done with relatively weak ion injection rates commensurate with those in the distant environment of comet Halley. In response to the linear temporal growth of the fluctuating magnetic field energy, the injected ions pitch-angle scatter toward isotropy in both the quasiparallel $\left(0^{\circ} \leq \alpha \leq 60^{\circ}\right)$ and quasiperpendicular $\left(60^{\circ}<\alpha \leq 90^{\circ}\right)$ regimes. For the injection of cometary oxygen ions the simulations show pitch angle scattering rates that are essentially independent of the solar wind ion/cometary ion relative speed, and increase as the square root of the injection rate. Furthermore, the oxygen ion pitch angle scattering rate at perpendicular injection is approximately twice that in the quasiparallel regime, in qualitative agreement with observations at comet Halley.
\end{abstract}

\section{Introduction}

Cometary ion distributions are often unstable in the solar wind, leading to the growth of fluctuating magnetic and electric fields. If, as is often the case, electromagnetic instabilities at frequencies below the cometary ion cyclotron frequency dominate, the resulting enhanced fluctuations pitch-angle scatter the cometary ions.

There have been several recent detailed studies of cometary ion pitch-angle scattering as observed in the distant environment of comet Halley. Neugebauer et al. [1989] showed that, outside the bow shock, cometary protons experienced much more rapid pitch-angle scattering than energy diffusion at all values of $\alpha$, the angle between the solar wind magnetic field and the solar wind flow velocity. Coates et al. $[1989,1990]$ showed that pitch-angle scattering of water group cometary ions in the distant Halley environment increased with reduced cometocentric distance and that, as for the protons, energy diffusion proceeded more slowly than pitch-angle scattering. Neugebauer et al. [1990] further quantified the cometary ion response and concluded that (1) the mean width of

Copyright 1991 by the American Geophysical Union.

Paper number 91GL01331

0094-8534/91/91GL-01331 the proton pitch-angle distribution remained relatively narrow and was nearly independent of cometocentric distance almost right up to the bow shock and (2) the mean width of the water group ion pitch angle clistributions increased both with increasing ion density (an expected result) and with increasing $\alpha$ (an unexpected result).

The linear theory of cometary ion instabilities has been reviewed by Gary [1991]; the current consensus is that in the quasiparallel regime $\left(0^{\circ} \leq \alpha \lesssim 60^{\circ}\right)$ electromagnetic ion/ion instabilities are most important, whereas electromagnetic ion cyclotron anisotropy instabilities dominate in the quasiperpendicular $\left(60^{\circ}<\alpha \leq 90^{\circ}\right)$ regime. The fact that the instability physics changes with $\alpha$ suggests that pitch-angle scattering should also be a function of this angle. However, although many quasilinear theories of cometary ion pitch-angle scattering have been published [Gaffey et al., 1988; Gaffey and Wu, 1989; Cravens, 1989; Ziebell et al., 1990; Ziebell and Yoon, 1990; Yoon et al., 1991; Ye and Cravens, 1991], none have furnished a description of how pitch-angle scattering rates vary as a function of $\alpha$.

The first published simulation study of the injected ion/solar wind interaction was that of Winske et al. [1984, 1985], who simulated fluctuation growth due to a tenuous ring-beam ion component of fixed density. Further fixedbeam simulations or test particle calculations of cometary ion pitch-angle scattering have been carried out by Wu et al., 1986; Price and Wu, 1987; Gaffey et al., 1988; and Ziebell et al., 1990. The computer simulations of Gary et al. [1986, 1988, 1989], Miller et al. [1991] and the present computations have also studied fluctuation growth induced by cometary ions. These simulations, however, differ from the fixed beam computations in that cometary jons are continuously injected at a constant, spatially uniform rate.

This paper and its companion, Miller et al.[1991], examine a topic which, to our knowledge, has not been addressed previously: the relationships between the rate of pitch-angle scattering induced by wave-particle interactions and two parameters, $\alpha$ and the cometary ion injection rate $\Lambda_{j}$. Thus we concentrate on relatively early time simulations, corresponding to rapid pitch-angle scattering; the later time simulation effects of ion thermalization and energization must be addressed elsewhere.

Our earlier research with cometary oxygen ions emphasized strong injection rates $\left(\Lambda_{j} \geq 2 \times 10^{-6} \Omega_{p}\right)$ which correspond to the exponential temporal growth regime at small $\alpha$ [Gary et al., 1988, 1989]. However, in this paper we deal only with the relatively weal ion injection 
rates $\left(\Lambda_{j} \leq 10^{-6} \Omega_{p}\right.$ where $\Omega_{p}$ is the proton cyclotron frequency) commensurate with the distant environment (i.e., beyond the bow shock) at Halley. Such rates correspond to the linear temporal growth regime of the fluctuating magnetic field energy as illustrated in Figure 1, which we believe is more appropriate for describing the distant Halley environment.

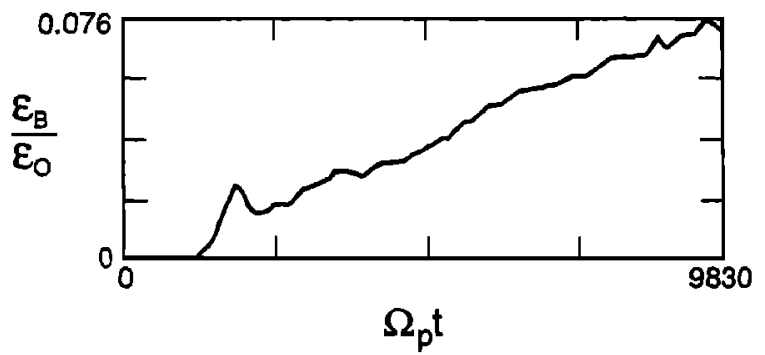

Fig. 1. The total fluctuating magnetic field energy density as a function of time for a representative computer simulation. Constant physical parameters of this simulation included: $m_{b}=16 m_{s w}, v_{0}=6 v_{A}, \alpha=0^{\circ}$, and $\Lambda_{O^{+}}=2.0 \times 10^{-7} \Omega_{p}$. The initial physical parameters included: $T_{e}=T_{s w}, \beta_{s w}=1.0$, and $v_{A} / c=10^{-4}$. This simulation was run at $\mathbf{k} \times \mathbf{B}_{\mathbf{0}}=0$, and used 40000 solar wind ions, a simulation time step $\Omega_{p} \Delta t=1.60$, and a simulation box size $L \omega_{s w} / c=2400$ divided into 128 cells. Unless explicitly varied or stated to be otherwise, parameters in all runs depicted in the following figures are as stated in this caption.

\section{Definitions}

We consider a three component plasma: a relatively tenuous injected ion component which we often denote by subscript "b" (for beam), the more dense solar wind protons ("sw") and electrons ("e"). The subscript "p" represents protons and " $\mathrm{O}^{+}$" denotes oxygen ions. Unless otherwise noted, our notation and definitions are identical to those in Gary et al., [1989].

The background magnetic field is uniform and $\mathbf{B}_{0}=$ $\hat{\mathbf{z}} B_{0}$. The pitch-angle $\theta_{i}$ of each ion $i$ is obtained from

$$
\cos \theta_{i}=\frac{v_{z i}}{\sqrt{v_{x i}^{2}+v_{y i}^{2}+v_{z i}^{2}}} .
$$

Thus the average pitch-angle of the $j$ th component ions in our simulations is

$$
\langle\theta\rangle_{j}=\frac{\sum_{i} \theta_{i}}{N_{j}}
$$

where the numerator represents the summation over the pitch-angles of the jth component ions and $N_{j}$ is the total number of $j$ th component ions in the simulation at the time of interest. Given this average pitch-angle, we then follow Coates et al. [1989] and Neugebauer et al. [1990] and define the average width of the pitch-angle distribution of the $j$ th component as

$$
<\theta>_{j}=\frac{\sum_{i}\left|\theta_{i}-<\theta>_{j}\right|}{N_{j}}
$$

The hybricl simulation code used here is based upon the code of Winske and Leroy [1984; 1985], in which the ions are treated as discrete superparticles and the electrons are taken as a massless, charge-neutralizing fluid. The interactions between the ions and the electric and magnetic fields are calculated self-consistently, and the zero-current condition is satisfied. The simulation is onedimensional in space but the particle velocities and electromagnetic fields are three-dimensional vectors. Periodic boundary conditions for both particles and fields are used.

The solar wind protons are constant in number and at $t=0$ are distributed uniformly in space with a zero drift Maxwellian velocity distribution. The $j$ th species cometary ions are introduced into the simulation at a constant rate $\Lambda_{j} \equiv\left(d n_{j} / d t\right) / n_{s} w$, randomly in space, and with constant initial velocity $\mathbf{v}_{0}=\hat{\mathbf{y}} v_{0} \sin \alpha+\hat{\mathbf{z}} v_{0} \cos \alpha$, thus simulating the ionization of cometary neutrals and the subsequent appearance of new cold ions.

\section{Computer Simulations}

We consider the average width of the pitcli-angle distribution defined by Equation (1). Although this quantity is not readily extracted from analytic theory, it exlibits non-trivial temporal variation in both the quasiparallel and quasiperpendicular regimes. For an isotropic distribution, $\langle\Theta\rangle_{b}=(\pi-2) / 2=32.70^{\circ}$. Thus we define

$$
<\Theta^{\prime}(t)>_{b} \equiv \frac{\pi-2}{2}-<\Theta(t)>_{b}
$$

which is plotted for a representative simulation in Figure 2. Since this quantity exhibits a reasonable approximation to a straight line when plotted on a semi-log scale not only here but in many of our simulations, we define the quantity $\nu_{\Theta}$ througli the expression

$$
<\Theta^{\prime}(t)>_{b}=\frac{\pi-2}{2} \exp \left(-\nu_{\Theta} t\right)
$$

Thus $\nu_{\Theta}$ represents a time-averaged pitch-angle scattering frequency.

We carried out a number of computer simulations using cometary protons. For injection rates commensurate with those in the distant environment at Halley, these

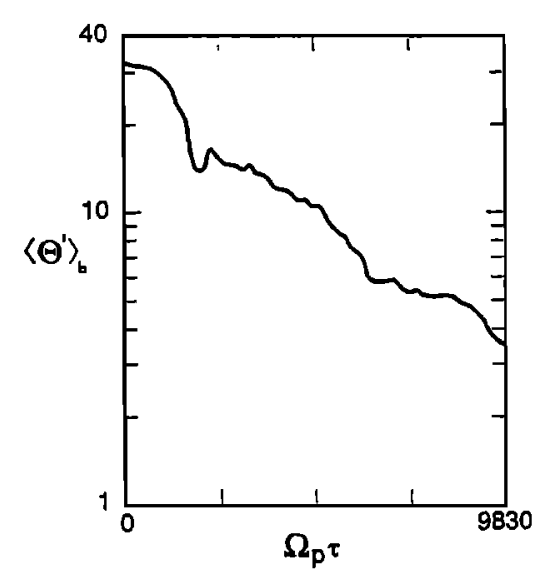

Fig. 2. Plot of $\left\langle\Theta^{\prime}\right\rangle_{b}$ as a function of time in the simulation of Figure 1. 

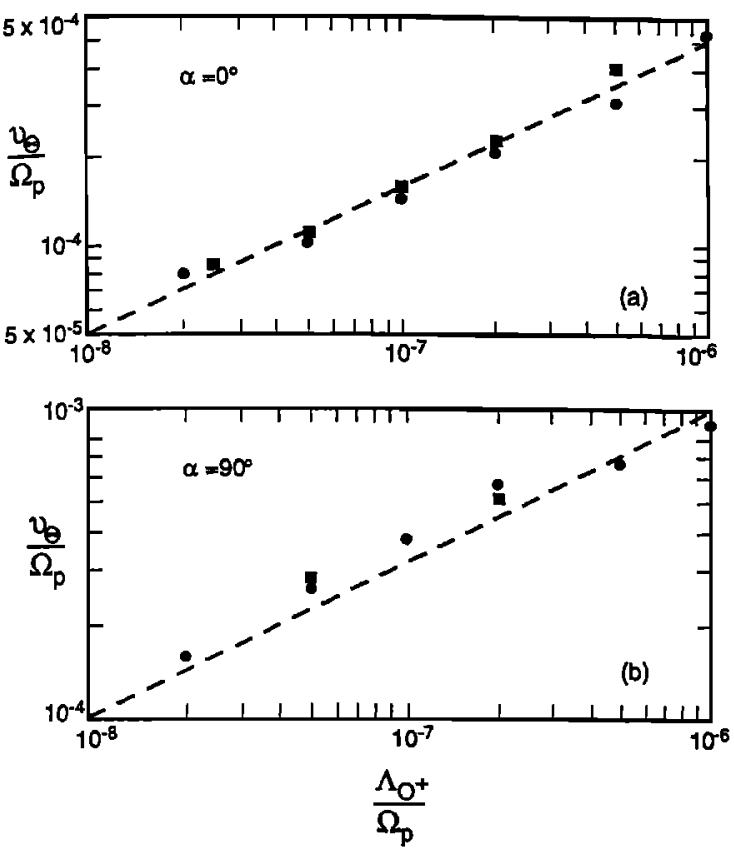

Fig. 3. The pitch angle scattering frequency $\nu_{\Theta}$ as a function of the cometary ion injection rate. Panel (a) corresponds to $\alpha=0^{\circ}$; the dashed line represents the scaling of Equation (3). Panel (b) corresponds to $\alpha=90^{\circ}$; the dashed line represents the scaling of Equation (4). Here and in Figure 4, the round dots represent results from simulations at $v_{0}=6 v_{A}$ and $L \omega_{s w} / c=2400$; the square dots correspond to simulations with $v_{o}=10 v_{A}$ and $L \omega_{s w} / c=4000$.

runs exhibited very weak pitcli-angle scattering at quasiparallel injection. Because this scattering was weak, we were unable to obtain useful scaling relations for proton injection; we note, however, that these results are in agreement with the observations of weak cometary proton pitch-angle scattering by Neugebauer et al.[1989 and 1990].

We executed a larger number of computer simulations using cometary oxygen ions; we here summarize the results of these simulations. Figure 3(a) shows the pitchangle scattering frequencies obtained from oxygen ion injection simulations as a function of the injection rate $\Lambda_{O+}$ at $\alpha=0^{\circ}$. The dashed line represents the empirical scaling relation

$$
\frac{\nu_{\Theta}}{\Omega_{p}} \simeq \frac{1}{2} \sqrt{\frac{\Lambda_{O+}}{\Omega_{p}}} \quad \alpha=0^{\circ}
$$

Since simulations at $v_{o}=6 v_{A}, 8 v_{A}$ and $10 v_{A}$ exhibit very similar pitch-angle scattering frequencies, we conclude this scaling is independent of injection speed over this range of $v_{o} / v_{A}$. Figure $3(\mathrm{~b})$ illustrates the pitch-angle scattering frequencies from oxygen ion injection simulations as a function of the injection rate at $\alpha=90^{\circ}$. In this case, the empirical scaling relation for this range of injection rates and $6 v_{A} \leq v_{o} \leq 10 v_{A}$ is

$$
\frac{\nu_{\Theta}}{\Omega_{p}} \simeq \sqrt{\frac{\Lambda_{O+}}{\Omega_{p}}} \quad \alpha=90^{\circ}
$$

Figure 4 shows the pitch-angle scattering frequency from a series of simulations in which all physical parameters except $\alpha$ were held constant. Here $\nu_{\Theta}$ is essentially independent of $\alpha$ out to $\alpha=60^{\circ}$, then shows an increase to about twice the quasiparallel value as $\alpha$ approaches the perpendicular. This result is in qualitative agreement witl the increase in pitch-angle scattering of water group ions with $\alpha$ observed by Neugebauer et al. [1990]. Since we have previously shown that the rate of fluctuating magnetic field energy generally decreases as $\alpha$ increases [Gary et al., 1989], the increase in scattering with $\alpha$ cannot be due to increased power in the fluctuations.

\section{Summary}

We have used a fully self-consistent hybrid computer simulation to study wave-particle interactions stimulated by injected ions in distant cometary environments. We find cometary proton pitch-angle scattering is relatively weak, in agreement with observations at comet Halley. For the injection of cometary oxygen ions our simulations show pitcli-angle scattering rates that increase as the square root of the injection rate. The oxygen ion pitch-angle scattering rates are roughly twice as large at perpendicular injection as they are at quasiparallel injection, again in qualitative agreement with observations.

Our results show some similarities with the results from recent quasilinear theories of cometary ion pitch angle scattering. For example, our Equation (2) approximately corresponds to Eq. (32) of Gaffey et al. [1988]. However, Gaffey and Wu [1989] expect water group pitch angle scattering to be weaker than proton scattering, and Ziebell et al. [1990] imply no seattering through perpendicular pitch angles in the limit of weak fluctuating fields, results in apparent contrast to our simulations. One possible source of these differences is that most recent theories use the assumption of constant fluctuating magnetic field amplitude, whereas the temporal change of fields is an intrinsic element of our simulations and, we believe, the cometary environment.

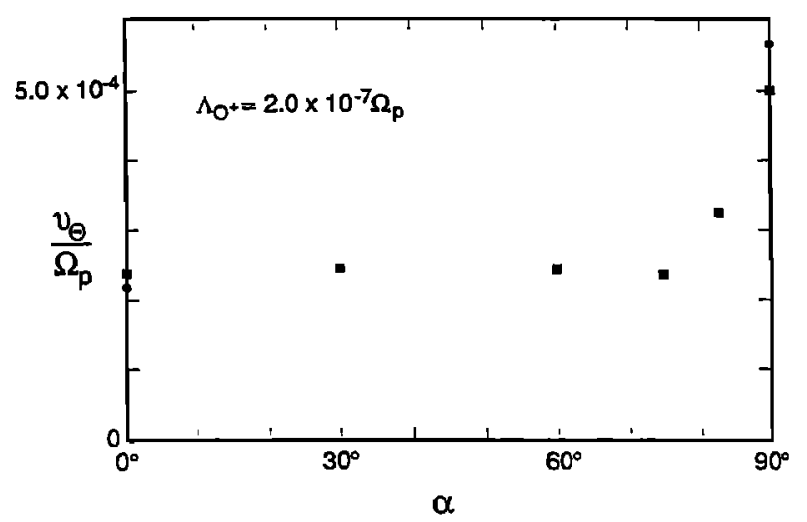

Fig. 4. The pitch-angle scattering frequency $\nu_{\Theta}$ as a function of $\alpha$, the angle between the solar wind velocity and the interplanetary magnetic field, at $\Lambda_{O+}=2.0 \times 10^{-7} \Omega_{p}$. 
Acknowledgments. The authors acknowledge useful discussions with Andrew Coates, Karen Flammer, Martin Lee, Marcia Neugebauer, and Nick Omidi. This work was performed under the auspices of the U.S. Department of Energy and was supported by the DOE Office of Basic Energy Sciences, Geosciences, and the Space Physics Research and Analysis Support Program of the National Aeronautics and Space Administration. The work of RHM at the University of Michigan was supported under NASA grants NAGW-2162 and NAGW-1366.

\section{References}

Coates, A. J., et al., Velocity-space diffusion of pickup ions from the water group at Comet Halley, J. Geophys. Res., 94, 9983, 1989; Correction, J. Geophys. Res., 95, 4343, 1990.

Coates, A. J., et al., Bulk properties and velocity distributions of water group ions at comet Halley: Giotto measurements, J. Geophys. Res., 95, 10249, 1990.

Cravens, T. E., Test particle calculations of pick-up ions in the vicinity of comet Giacobini-Zinner, Planet. Space Sci., 97, 1169, 1989.

Gaffey, J. D., Jr., D. Winske, and C. S. Wu, Time scales for formation and spreading of velocity shells of pickup ions in the solar wind, J. Geophys. Res., 93, 5470, 1088.

Gaffey, J. D., and C. S. Wu, Distribution function of continuously created newborn and pickup ions in outer cometary exospheres, J. Geophys. Res., 94, 8685, 1989.

Gary, S. P., Electromagnetic ion/ion instabilities and their consequences in space plasmas: a review, Space Sci. Revs., 56, 373, 1991.

Gary, S. P., S. Hinata, C. D. Madland, and D. Winske, The development of shell-like distributions from newborn cometary ions, Geophys. Res. Lett., 19, 1364, 1986.

Gary, S. P., C. D. Madland, N. Omidi, and D. Winske, Computer simulations of two-pickup-ion instabilities in a cometary environment, J. Geophys. Res., 93, 9584, 1988.

Gary, S. P., K. Akimoto, and D. Winske, Computer simulations of cometary-ion/ion instabilities and wave growth, J. Geophys. Res., 94, 3513, 1989.

Miller, R. H., S. P. Gary, D. Winske, and T. I. Gombosi, Pitch angle scattering of cometary ions into monospherical and bispherical distributions, Geophys. Res. Lett., 18, this issue, 1991.

Neugebauer, M., et al., The velocity distribution of cometary protons picked up by the solar wind, J. Geophys. Res., 94, 5227, 1989.
Neugebauer, M., A. J. Coates, and F. M. Neubauer, Comparison of picked-up protons and water-group ions upstream of comet Halley's bow shock, J. Geophys. Res., 95, 18745, 1990.

Price, C. P., and C. S. Wu, The influence of strong hydromagnetic turbulence on newborn cometary ions, Geophys. Res. Lett., 14, 856, 1987.

Winske, D. and M. M. Leroy, Diffuse ions produced by electromagnetic ion beam instabilities, $J$. Geophys. Res., 89, 2673, 1984.

Winske, D., and M. M. Leroy, Hybrid simulation techniques applied to the Earth's bow shock, in Computer Simulations of Space Plasmas - Selected Lectures at the First ISSS, edited by H. Matsumoto and T. Sato, p. 568, Terra Publishers, Reidel, Dordrecht, 1985.

Winske, D., C. S. Wu, Y. Y. Li, and S. Y. Guo, Collective capture of released lithium ions in the solar wind, $J$. Geophys. Res., 89, 7327, 1984.

Winske, D., C. S. Wu, Y. Y. Li, Z. Z. Mou, and S. Y. Guo, Coupling of newborn ions to the solar wind by electromagnetic instabilities and their interaction with the bow sliock, J. Geophys. Res., 90, 2713, 1985.

Wu, C. S., D. Winske, and J. D. Gaffey, Rapid pickup of cometary ions due to strong nagnetic turbulence, Geophys. Res. Lett., 13, 865, 1986.

Ye, G., and T. E. Cravens, Combined energy and pitch angle diffusion of pickup ions at comet Halley, J. Geophys. Res., 96, 5479, 1991.

Yoon, P. H., L. F. Ziebell, and C. S. Wu, Self-consistent pitch angle diffusion of newborn ions, $J$. Geophys. Res., 96, 5469, 1991.

Ziebell, L. F., P. H. Yoon, C. S. Wu, and D. Winske, Pitch angle diffusion of newborn ions due to intrinsic turbulence in the solar wind, J. Geophys. Res., 95, 17075, 1990.

Ziebell, L. F., and P. H. Yoon, Pitch angle and velocity diffusions of newborn ions by turbulence in the solar wind, J. Geophys. Res., 95, 21203, 1990.

S. P. Gary and D. Winske, M.S. D438, Los Alamos National Laboratory, Los Alamos, NM 87545.

R. H. Miller, Department of Atmospheric, Oceanic and Space Sciences, University of Michigan, Ann Arbor, MI 48109 .

(Received March 18, 1991; revised April 25, 1991; accepted May 10, 1991) 